American J. of Engineering and Applied Sciences 2 (2): 501-514, 2009

ISSN 1941-7020

(C) 2009 Science Publications

\title{
Femtotechnology: Nuclear Matter with Fantastic Properties
}

\author{
A.A. Bolonkin \\ Department Aviation/Aerospace, 1310 Avenue R, \\ No. 6-F, Brooklyn New York, 11229, USA
}

\begin{abstract}
Problem statement: At present the term 'nanotechnology' is well known-in its' ideal form, the flawless and completely controlled design of conventional molecular matter from molecules or atoms. Such a power over nature would offer routine achievement of remarkable properties in conventional matter and creation of metamaterials where the structure not the composition brings forth new powers of matter. But even this yet unachieved goal is not the end of material science possibilities. The author herein offers the idea of design of new forms of nuclear matter from nucleons (neutrons, protons), electrons and other nuclear particles. Approach: The researcher researches the nuclear forces. He shows these force may be used for design the new nuclear matter from protons, neutrons, electrons and other nuclear particles. Results: Author shows this new 'AB-Matter' has extraordinary properties (for example, tensile strength, stiffness, hardness, critical temperature, superconductivity, supertransparency and zero friction.), which are up to millions of times better than corresponding properties of conventional molecular matter. He shows concepts of design for aircraft, ships, transportation, thermonuclear reactors, constructions and so on from nuclear matter. These vehicles will have unbelievable possibilities (e.g., invisibility, ghost-like penetration through any walls and armor, protection from nuclear bomb explosions and any radiation flux). Conclusion: People may think this fantasy. But fifteen years ago most people and many scientists thought-nanotechnology is fantasy. Now many groups and industrial labs, even startups, spend hundreds of millions of dollars for development of nanotechnological-range products (precise chemistry, patterned atoms, catalysts and meta-materials) and we have nanotubes (a new material which does not exist in Nature!) and other achievements beginning to come out of the pipeline in prospect. Nanotubes are stronger than steel by a hundred times-surely an amazement to a 19th Century observer if he could behold them. Nanotechnology, in near term prospect, operates with objects (molecules and atoms) having the size in nanometer $\left(10^{-9} \mathrm{~m}\right)$. The researcher here outlines perhaps more distant operations with objects (nuclei) having size in the femtometer range, $\left(10^{-15} \mathrm{~m}\right.$, millions of times less smaller than the nanometer scale). The name of this new technology is femtotechnology.
\end{abstract}

Key words: Femtotechnology, nuclear matter, artificial AB-Matter, superstrength matter, superthermal resistance, invisible matter, super-protection from nuclear explosion and radiation

\section{INTRODUCTION}

Brief information concerning the atomic nucleus ${ }^{[1,2]}$ : Atoms are the smallest (size is about some $10^{-8} \mathrm{~m}$ ) neutral particles into which matter can be divided by chemical reactions. An atom consists of a small, heavy nucleus surrounded by a relatively large, light cloud of electrons. Each type of atom corresponds to a specific chemical element. To date, 117 elements have been discovered (atomic numbers 1-116 and 118) and the first 111 have received official names. The well-known periodic table provides an overview. Atoms consist of protons and neutrons within the nucleus. Within these particles, there are smaller particles still which are then made up of even smaller particles still.
Molecules are the smallest particles into which a non-elemental substance can be divided while maintaining the physical properties of the substance. Each type of molecule corresponds to a specific chemical compound. Molecules are a composite of two or more atoms.

Atoms contain small (size is about some $10^{-15} \mathrm{~m}$ ) nuclei and electrons orbit around these nuclei. The nuclei of most atoms consist of protons and neutrons, which are therefore collectively referred to as nucleons. The number of protons in a nucleus is the atomic number and defines the type of element the atom forms. The number of neutrons determines the isotope of an element. For example, the carbon-12 isotope has 6 protons and 6 neutrons, while the carbon-14 isotope has 6 protons and 8 neutrons. 
While bound neutrons in stable nuclei are stable, free neutrons are unstable; they undergo beta decay with a lifetime of just under $15 \mathrm{~min}$. Free neutrons are produced in nuclear fission and fusion. Dedicated neutron sources like research reactors and spallation sources produce free neutrons for the use in irradiation and in neutron scattering experiments.

Outside the nucleus, free neutrons are unstable and have a mean lifetime of $885.7 \pm 0.8 \mathrm{sec}$, decaying by emission of a negative electron and antineutrino to become a proton:

$$
\mathrm{n}^{0} \rightarrow \mathrm{p}^{+}+\mathrm{e}^{-}+\mathrm{v}_{\mathrm{e}}
$$

This decay mode, known as beta decay, can also transform the character of neutrons within unstable nuclei. Bound inside a nucleus, protons can also transform via inverse beta decay into neutrons. In this case, the transformation occurs by emission of a positron (antielectron) and a neutrino (instead of an antineutrino):

$$
\mathrm{p}^{+} \rightarrow \mathrm{n}^{0}+\mathrm{e}^{+}+v_{\mathrm{e}}
$$

The transformation of a proton to a neutron inside of a nucleus is also possible through electron capture:

$$
\mathrm{p}^{+}+\mathrm{e}^{-} \rightarrow \mathrm{n}^{0}+v_{\mathrm{e}}
$$

Positron capture by neutrons in nuclei that contain an excess of neutrons is also possible, but is hindered because positrons are repelled by the nucleus and quickly annihilate when they encounter negative electrons. When bound inside of a nucleus, the instability of a single neutron to beta decay is balanced against the instability that would be acquired by the nucleus as a whole if an additional proton were to participate in repulsive interactions with the other protons that are already present in the nucleus. As such, although free neutrons are unstable, bound neutrons are not necessarily so. The same reasoning explains why protons, which are stable in empty space, may transform into neutrons when bound inside of a nucleus.

A thermal neutron is a free neutron that is Boltzmann distributed with $\mathrm{kT}=0.024 \mathrm{eV}\left(4.0 \times 10^{-21} \mathrm{~J}\right)$ at room temperature. This gives characteristic (not average, or median) speed of $2.2 \mathrm{~km} \mathrm{sec}^{-1}$. Four forces active between particles: strong interaction, weak interacting, charge force (Coulomb force) and gravitation force. The strong interaction is the most strong force in short nuclei distance, the gravitation is very small into atom. Beta decay and electron capture are types of radioactive decay and are both governed by the weak interaction.

Basic properties of the nuclear force: The nuclear force is only felt among hadrons. In particle physics, a hadron is a bound state of quarks (particles into nucleous). Hadrons are held together by the strong force, similarly to how atoms are held together by the electromagnetic force. There are two subsets of hadrons: baryons and mesons; the most well known baryons are protons and neutrons.

At much smaller separations between nucleons the force is very powerfully repulsive, which keeps the nucleons at a certain average separation. Beyond about 1.7 femtometer (fm) separation, the force drops to negligibly small values.

At short distances, the nuclear force is stronger than the Coulomb force; it can overcome the Coulomb repulsion of protons inside the nucleus. However, the Coulomb force between protons has a much larger range and becomes the only significant force between protons when their separation exceeds about $2.5 \mathrm{fm}$. The nuclear force is nearly independent of whether the nucleons are neutrons or protons. This property is called charge independence. It depends on whether the spins of the nucleons are parallel or antiparallel and has a noncentral or tensor component. This part of the force does not conserve orbital angular momentum, which is a constant of motion under central forces.

The nuclear force (or nucleon-nucleon interaction or residual strong force) is the force between two or more nucleons. It is responsible for binding of protons and neutrons into atomic nuclei. To a large extent, this force can be understood in terms of the exchange of virtual light mesons, such as the pions. Sometimes the nuclear force is called the residual strong force, in contrast to the strong interactions which are now understood to arise from Quantum Chromo-Dynamics (QCD). This phrasing arose during the 1970s when QCD was being established. Before that time, the strong nuclear force referred to the inter-nucleon potential. After the verification of the quark model, strong interaction has come to mean QCD.

A subatomic particle is an elementary or composite particle smaller than an atom. Particle physics and nuclear physics are concerned with the study of these particles, their interactions and non-atomic matter. Elementary particles are particles with no measurable internal structure; that is, they are not composed of other particles. They are the fundamental objects of quantum field theory. Many families and sub-families of elementary particles exist. Elementary particles are classified according to their spin. Fermions have half- 
integer spin while bosons have integer spin. All the particles of the Standard Model have been observed, with the exception of the Higgs boson.

Subatomic particles include the atomic constituents electrons, protons and neutrons. Protons and neutrons are composite particles, consisting of quarks. A proton contains two up quarks and one down quark, while a neutron consists of one up quark and two down quarks; the quarks are held together in the nucleus by gluons. There are six different types of quark in all ('up', 'down', 'bottom', 'top', 'strange' and 'charm'), as well as other particles including photons and neutrinos which are produced copiously in the sun. Most of the particles that have been discovered are encountered in cosmic rays interacting with matter and are produced by scattering processes in particle accelerators. There are dozens of known subatomic particles.

Degenerate matter: Degenerate matter is matter which has such very high density that the dominant contribution to its pressure rises from the Pauli exclusion principle. The pressure maintained by a body of degenerate matter is called the degeneracy pressure and arises because the Pauli principle forbids the constituent particles to occupy identical quantum states. Any attempt to force them close enough together that they are not clearly separated by position must place them in different energy levels. Therefore, reducing the volume requires forcing many of the particles into higher-energy quantum states. This requires additional compression force and is manifest as a resisting pressure.

Imagine that there is a plasma and it is cooled and compressed repeatedly. Eventually, we will not be able to compress the plasma any further, because the Exclusion Principle states that two particles cannot be in the exact same place at the exact same time. When in this state, since there is no extra space for any particles, we can also say that a particle's location is extremely defined. Therefore, since (according to the Heisenberg Uncertainty Principle):

$$
\Delta \mathrm{p} \Delta \mathrm{x}=\hbar / 2
$$

Where:

$\Delta \mathrm{p}=$ The uncertainty in the particle's momentum $\Delta \mathrm{x}=$ The uncertainty in position

Then we must say that their momentum is extremely uncertain since the molecules are located in a very confined space. Therefore, even though the plasma is cold, the molecules must be moving very fast on average. This leads to the conclusion that if you want to compress an object into a very small space, you must use tremendous force to control its particles' momentum.

Unlike a classical ideal gas, whose pressure is proportional to its temperature:

$$
\mathrm{PV}=\mathrm{NkT}
$$

Where:

$\mathrm{P}=$ Pressure

$\mathrm{V}=$ The volume

$\mathrm{N}=$ The number of particles (typically atoms or molecules)

$\mathrm{k}=$ Boltzmann's constant

$\mathrm{T}=$ Temperature), the pressure exerted by degenerate matter depends only weakly on its temperature

In particular, the pressure remains nonzero even at absolute zero temperature. At relatively low densities, the pressure of a fully degenerate gas is given by:

$$
\mathrm{P}=\mathrm{Kn}^{5 / 3}
$$

where, $\mathrm{K}$ depends on the properties of the particles making up the gas. At very high densities, where most of the particles are forced into quantum states with relativistic energies, the pressure is given by:

$$
\mathrm{P}=\mathrm{K}^{\prime} \mathrm{n}^{4 / 3}
$$

where, $\mathrm{K}^{\prime}$ again depends on the properties of the particles making up the gas.

Degenerate matter still has normal thermal pressure, but at high densities the degeneracy pressure dominates. Thus, increasing the temperature of degenerate matter has a minor effect on total pressure until the temperature rises so high that thermal pressure again dominates total pressure.

Exotic examples of degenerate matter include neutronium, strange matter, metallic hydrogen and white dwarf matter. Degeneracy pressure contributes to the pressure of conventional solids, but these are not usually considered to be degenerate matter as a significant contribution to their pressure is provided by the interplay between the electrical repulsion of atomic nuclei and the screening of nuclei from each other by electrons allocated among the quantum states determined by the nuclear electrical potentials. In metals it is useful to treat the conduction electrons alone as a degenerate, free electron gas while the majority of the electrons are regarded as occupying bound quantum states. This contrasts with the case of the degenerate matter that forms the body of a white dwarf where all the electrons would be treated as occupying free particle momentum states. 


\section{MATERIALS AND METHODS}

Pauli principle: The Pauli exclusion principle is a quantum mechanical principle formulated by Wolfgang Pauli in 1925. It states that no two identical fermions may occupy the same quantum state simultaneously. A more rigorous statement of this principle is that, for two identical fermions, the total wave function is antisymmetric. For electrons in a single atom, it states that no two electrons can have the same four quantum numbers, that is, if $n, 1$ and $m_{l}$ are the same, $m_{s}$ must be different such that the electrons have opposite spins. In relativistic quantum field theory, the Pauli principle follows from applying a rotation operator in imaginary time to particles of half-integer spin. It does not follow from any spin relation in non-relativistic quantum mechanics. The Pauli exclusion principle is one of the most important principles in physics, mainly because the three types of particles from which ordinary matter is made-electrons, protons and neutrons-are all subject to it; consequently, all material particles exhibit spaceoccupying behavior. The Pauli exclusion principle underpins many of the characteristic properties of matter from the large-scale stability of matter to the existence of the periodic table of the elements. Particles with anti-symmetric wave functions are called fermions-and obey the Pauli exclusion principle. Apart from the familiar electron, proton and neutron, these include neutrinos and quarks (from which protons and neutrons are made), as well as some atoms like helium3. All fermions possess "half-integer spin", meaning that they possess an intrinsic angular momentum whose value is $\hbar=\mathrm{h} / 2 \pi$ (Planck's constant divided by $2 \pi$ ) times a half-integer $(1 / 2,3 / 2,5 / 2)$. In the theory of quantum mechanics, fermions are described by "antisymmetric states", which are explained in greater detail in the theory on identical particles. Particles with integer spin have a symmetric wave function and are called bosons; in contrast to fermions, they may share the same quantum states. Examples of bosons include the photon, the Cooper pairs responsible for superconductivity and the $\mathrm{W}$ and $\mathrm{Z}$ bosons.

A more rigorous proof was provided by Freeman Dyson and Andrew Lenard ${ }^{[11]}$, who considered the balance of attractive (electron-nuclear) and repulsive (electron-electron and nuclear-nuclear) forces and showed that ordinary matter would collapse and occupy a much smaller volume without the Pauli principle. Neutrons are the most "rigid" objects known-their Young modulus (or more accurately, bulk modulus) is 20 orders of magnitude larger than that of diamond. For white dwarfs the degenerate particles are the electrons while for neutron stars the degenerate particles are neutrons. In degenerate gas, when the mass is increased, the pressure is increased and the particles become spaced closer together, so the object becomes smaller. Degenerate gas can be compressed to very high densities, typical values being in the range of $10^{7} \mathrm{~g} \mathrm{~cm}^{-3}$. Preons are subatomic particles proposed to be the constituents of quarks, which become composite particles in preon-based models.

Neutron stars: A neutron star is a large gravitationallybound lump of electrically neutral nuclear matter, whose pressure rises from zero (at the surface) to an unknown value in the center.

A neutron star is a type of remnant that can result from the gravitational collapse of a massive star during a Type II, Type Ib or Type Ic supernova event. Such stars are composed almost entirely of neutrons, which are subatomic particles with zero electrical charge and roughly the same mass as protons.

A typical neutron star has a mass between 1.35 and about 2.1 solar masses, with a corresponding radius of about $12 \mathrm{~km}$ if the Akmal-PandharipandeRavenhall (APR) Equation Of State (EOS) is used. In contrast, the Sun's radius is about 60,000 times that. Neutron stars have overall densities predicted by the APR EOS of $3.7 \times 10^{17}\left(2.6 \times 10^{14}\right.$ times Solar density) to $5.9 \times 10^{17} \mathrm{~kg} \mathrm{~m}^{-3}\left(4.1 \times 10^{14}\right.$ times Solar density). Which compares with the approximate density of an atomic nucleus of $3 \times 10^{17} \mathrm{~kg} \mathrm{~m}^{-3}$. The neutron star's density varies from below $1 \times 10^{9} \mathrm{~kg} \mathrm{~m}^{-3}$ in the crust increasing with depth to above 6 or $8 \times 10^{17} \mathrm{~kg} \mathrm{~m}^{-3}$ deeper inside.

In general, compact stars of less than 1.44 solar masses, the Chandrasekhar limit, are white dwarfs; above 2-3 solar masses (the Tolman-OppenheimerVolkoff limit), a quark star might be created, however this is uncertain. Gravitational collapse will always occur on any star over 5 solar masses, inevitably producing a black hole. The gravitational field at the star's surface is about $2 \times 10^{11}$ times stronger than on Earth. The escape velocity is about $100,000 \mathrm{~km} \mathrm{sec}^{-1}$, which is about one third the speed of light. Such a strong gravitational field acts as a gravitational lens and bends the radiation emitted by the star such that parts of the normally invisible spectrum near the surface become visible.

The gravitational binding energy of a two solar mass neutron star is equivalent to the total conversion of one solar mass to energy (From the law of massenergy equivalence, $\mathrm{E}=\mathrm{mc}^{2}$ ). That energy was released during the supernova explosion. A neutron star is so dense that one teaspoon $(5 \mathrm{~mL})$ of its 
material would have a mass over $5 \times 10^{12} \mathrm{~kg}$. The resulting force of gravity is so strong that if an object were to fall from just one meter high it would hit the surface of the neutron star at 2 thousand $\mathrm{km} \mathrm{sec}^{-1}$, or 4.3 million miles $\mathrm{h}^{-1}$.

The Equation Of State (EOS) for a Neutron star is still not known as of 2008. On the basis of current models, the matter at the surface of a neutron star is composed of ordinary atomic nuclei as well as electrons.

\section{Innovations and computations:}

Short information about atom and nuclei: Conventional matter consists of atoms and molecules. Molecules are collection of atoms. The atom contains a nucleus with proton (s) and usually neutrons (Except for Hydrogen-1) and electrons revolve around this nucleus. Every particle may be characterized by parameters as mass, charge, spin, electric dipole, magnetic moment. There are four forces active between particles: Strong interaction, weak interaction, electromagnetic charge (Coulomb) force and gravitational force. The nuclear force dominates at distances up to $2 \mathrm{fm}$ (femto, $1 \mathrm{fm}=10^{-15} \mathrm{~m}$ ). They are hundreds of times more powerful than the charge (Coulomb force and million-millions of times more than gravitational force. Charge (Coulomb) force is effective at distances over $2 \mathrm{fm}$. Gravitational force is significant near and into big masses (astronomical objects such as planets, stars, white dwarfs, neutron stars and black holes). Strong force is so overwhelmingly powerful that it forces together the positively charged protons, which would repel one from the other and fly apart without it. The strong force is key to the relationship between protons, neutrons and electrons. They can keep electrons into or near nuclei. Scientists conventionally take into attention only of the strong force when they consider the nuclear and near nuclear size range, for the other forces on that scale are negligible by comparison for most purposes.

Strong nuclear forces are anisotropic (non spherical, force distribution not the same in all directions equally), which means that they depend on the relative orientation of the nucleus.

Typical nuclear energy (force) is presented in Fig. 1. When it is positive the nuclear force repels the other atomic particles (protons, neutrons, electrons). When nuclear energy is negative, it attracts them up to a distance of about $2 \mathrm{fm}$. The value $r_{0}$ usually is taken as radius of nucleus. The computation of strong nuclear force-interaction energy of one nucleus via specific density of one nucleus in given point-is show in Fig. 2. The solid line is as computed by Berkner's method ${ }^{[7]}$ with 2 correlations, dotted line is computer generated with 3 correlations, square is experimental. Average interaction energy between to nucleus is about $8 \mathrm{MeV}$, distance where the attractive strong nuclear force activates is at about $1-1.2 \mathrm{fm}$.

AB-matter: In conventional matter made of atoms and molecules the nucleons (protons, neutrons) are located in the nucleus, but the electrons rotate in orbits around nucleus in distance in millions times more than diameter of nucleus. Therefore, in essence, what we think of as solid matter contains a-relatively!-'gigantic' vacuum (free space) where the matter (nuclei) occupies but a very small part of the available space.

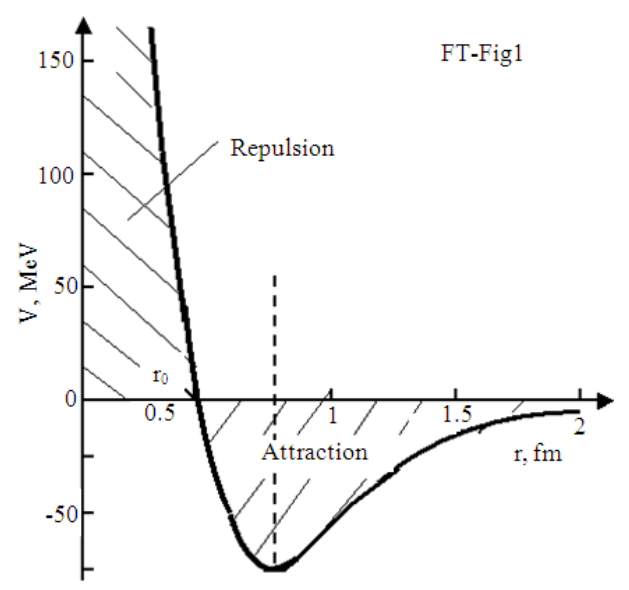

Fig. 1: Typical nuclear force of nucleus. When nucleon is at distance of less than $1.8 \mathrm{fm}$, it is attracted to nucleus. When nucleon is very close, it is repulsed from nucleus ${ }^{[7]}$

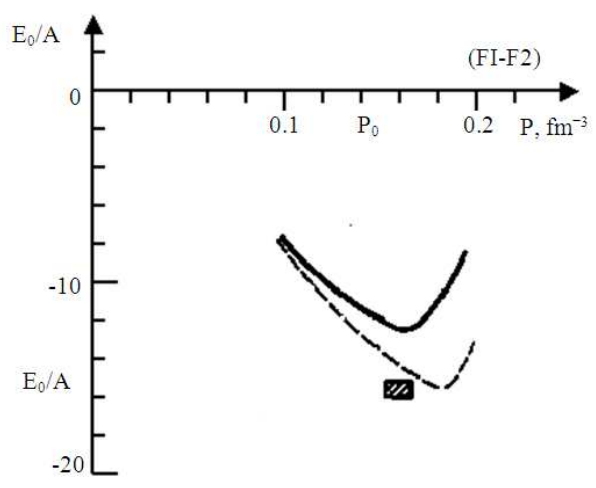

Fig. 2: Connection (interaction) energy of one nucleon via specific density of one nucleon in given point. Firm line is computed by Berkner's method with 2 correlations, dotted line is computer with 3 correlations, square is experiment $^{[7]}$ 
Despite this unearthly emptiness, when you compress this (normal, non-degenerate) matter the electrons located in their orbits repel atom from atom and resist any great increase of the matter's density. Thus it feels solid to the touch.

The form of matter containing and subsuming all the atom's particles into the nucleus is named degenerate matter. Degenerate matter found in white dwarfs, neutron stars and black holes. Conventionally this matter in such large astronomical objects has a high temperature (as independent particles!) and a high gravity adding a forcing, confining pressure in a very massive celestial objects. In nature, degenerate matter exists stably (as a big lump) to our knowledge only in large astronomical masses (include their surface where gravitation pressure is zero) and into big nuclei of conventional matter.

Our purpose is to design artificial small masses of synthetic degenerate matter in form of an extremely thin strong thread (fiber, filament, string), round bar (rod), tube, net (dense or non dense weave and mesh size) which can exist at Earth-normal temperatures and pressures. Note that such stabilized degenerate matter in small amounts does not exist in Nature as far as we know. Therefore I have named this matter AB-Matter. Just as people now design by the thousands variants of artificial materials (for example, plastics) from usual matter, we soon (historically speaking) shall create many artificial, designer materials by nanotechnology (for example, nanotubes: SWNTs (amchair, zigzag, ahiral), MWNTs (fullorite, torus, nanobut), nanoribbon (plate), buckyballs (ball), fullerene). Sooner or later we may anticipate development of femtotechnology and create such $\mathrm{AB}-\mathrm{Matter}$. Some possible forms of $\mathrm{AB}$ Matter are shown in Fig. 3. Offered technologies are below. The threads from AB-Matter are stronger by millions of times than normal materials. They can be inserted as reinforcements, into conventional materials, which serve as a matrix and are thus strengthened by thousands of times.

Some offered technologies for producing: AB-Matter. One method of producing AB-Matter may use the technology reminiscent of computer chips (Fig. 4). One side of closed box 1 is evaporation mask 2 . In the other size are located the sources of neutrons, charged nuclear particles (protons, charged nuclei and their connections) and electrons. Sources (guns) of charged particles have accelerators of particles and control their energy and direction. They concentrate (focus) particles, send particles (in beam form) to needed points with needed energy for overcoming the Coulomb barrier. The needed neutrons are received also from nuclear reactions and reflected by the containing walls.

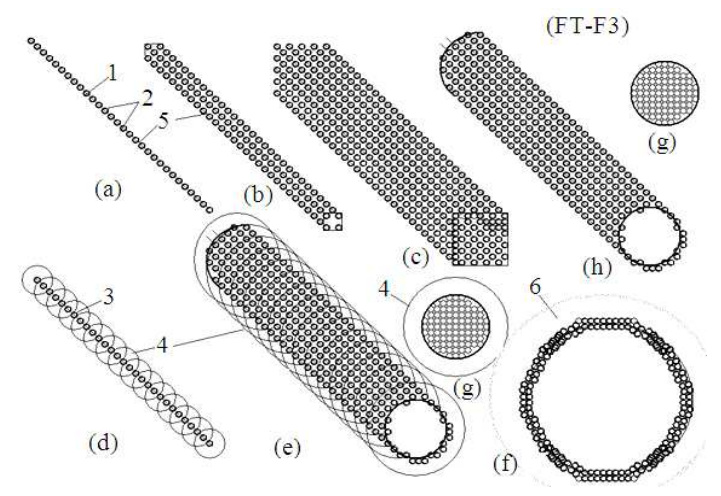

Fig. 3: Design of AB-Matter from nucleons (neutrons, protons) and electrons; (a): Linear one string (monofilament) (fiber, whisker, filament, thread); (b): Ingot from four nuclear monofilaments; (c): Multi-ingot from nuclear monofilament; (d): String made from protons and neutrons with electrons rotated around monofilament; (e): Single wall femto tube (SWFT) fiber with rotated electrons; (f): Cross-section of multi wall femto tube (MWFT) string; (g): Cross-section of rod; (h): Single Wall Femto Tube (SWFT) string with electrons inserted into AB-Matter. Notations: (1): Nuclear string; (2): Nucleons (neutrons, protons); (3): Protons; (4): Orbit of electrons; (5): Electrons; (6): Cloud of electrons around tube

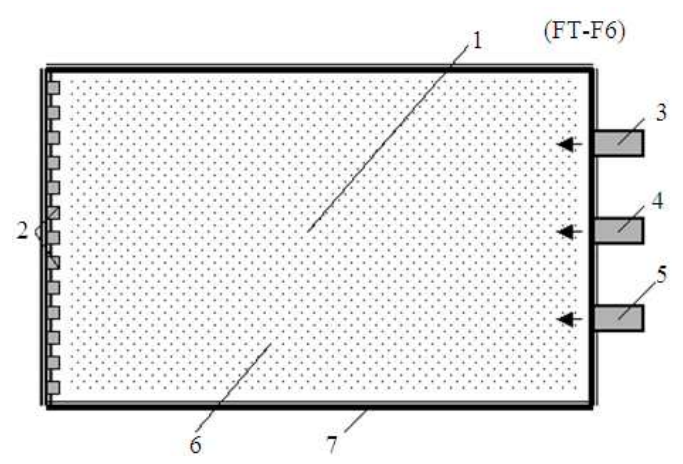

Fig. 4: Conceptual diagram for installation producing AB-Matter. Notations: (1): Installation; (2): AB-Matter (an extremely thin thread, round bar, rod, tube, net) and form mask; (3): Neutron source; (4): Source of charged particles (protons, charged nuclei), accelerator of charged particle, throttle control, beam control; (5): Source of electrons, accelerator of electrons, throttle control, beam control; (6): Cloud of particles; (7): Walls reflect the neutrons and utilize the nuclear energy 
Various other means are under consideration for generation of AB-Matter, what is certain however is that once the first small amounts have been achieved, larger and larger amounts will be produced with ever increasing ease. Consider for example, that once we have achieved the ability to make a solid AB-Matter film (a sliced plane through a solid block of AB-matter) and then developed the ability to place holes with precision through it one nucleon wide, a modified extrusion technique may produce $\mathrm{AB}$-Matter strings (thin fiber), by passage of conventional matter in gas, liquid or solid state through the AB-Matter matrix (mask). This would be a 'femto-die' as Joseph Friedlander of Shave Shomron, Israel, has labeled it. Re-assembling these strings with perfect precision and alignment would produce more AB-matter film; leaving deliberate gaps would reproduce the 'holes' in the initial 'femto-die'.

The developing of femtotechnology is easier, in one sense, than the developing of fully controllable nanotechnology because we have only three main particles (protons, neutrons, their ready combination of nuclei ${ }_{2} \mathrm{D},{ }_{3} \mathrm{~T},{ }_{4} \mathrm{He}$ and electrons) as construction material and developed methods of their energy control, focusing and direction.

Using the AB-matter: The simplest use of AB-Matter is strengthening and reinforcing conventional material by AB-Matter fiber. As it is shown in the 'Computation' section, AB-Matter fiber is stronger (has a gigantic ultimate tensile stress) than conventional material by a factor of millions of times, can endure millions degrees of temperature, don't accept any attacking chemical reactions. We can insert (for example, by casting around the reinforcement) $\mathrm{AB}$ Matter fiber (or net) into steel, aluminum, plastic and the resultant matrix of conventional material increases in strength by thousands of times-if precautions are taken that the reinforcement stays put! Because of the extreme strength disparity design tricks must be used to assure that the fibers stay 'rooted'. The matrix form of conventional artificial fiber reinforcement is used widely in current technology. This increases the tensile stress resistance of the reinforced matrix matter by typically 2-4 times. Engineers dream about a nanotube reinforcement of conventional matrix materials which might increase the tensile stress by 10-20 times, but nanotubes are very expensive and researchers cannot decrease its cost to acceptable values yet despite years of effort. Another way is using a construct of $\mathrm{AB}$ Matter as a continuous film or net (Fig. 5 b and d).

These forms of AB-Matter have such miraculous properties as invisibility, superconductivity, zero friction. The ultimate in camouflage, installations of a veritable Invisible World can be built from certain forms of $\mathrm{AB}-M a t t e r$ with the possibility of being also

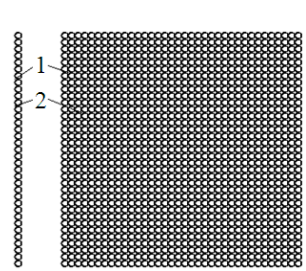

(a)

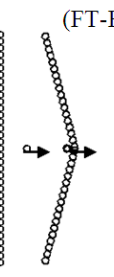

(c)

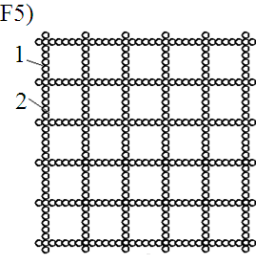

(d)
Fig. 5: Thin film from nuclear matter; (a): crosssection of a matter film from single strings (side view); (b): continuous film from nuclear matter; (c): $\mathrm{AB}$ film under blow from conventional molecular matter; (d): Net from single strings. Notations: 1: Nucleons; 2: Electrons inserted into AB-Matter; 3: Conventional atom

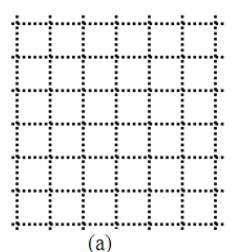

(a)

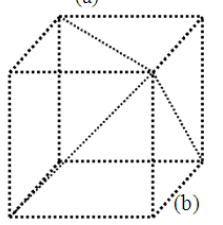

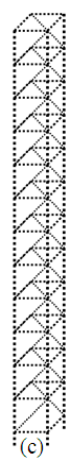

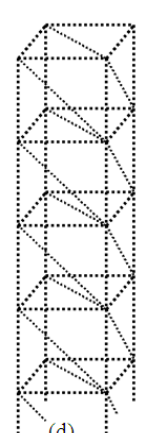

(d)

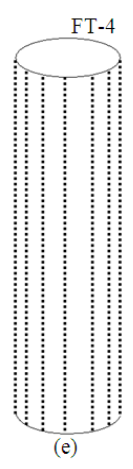

Fig. 6: Structures from nuclear strings. (a): Nuclear net (netting, gauze); (b): Primary cube from matter string; (c): Primary column from nuclear string; (d): Large column where elements made from primary columns; (e): Tubes from matter string or matter columns

interpenetable, literally allowing ghost-like passage through an apparently solid wall. Or the AB-Matter net (of different construction) can be designed as an impenetrable wall that even hugely destructive weapons cannot penetrate.

The AB-Matter film and net may be used for energy storage which can store up huge energy intensities and used also as rocket engines with gigantic impulse or weapon or absolute armor (see computation and application sections). Note that in the case of absolute armor, safeguards must be in place against buffering sudden accelerations; g-force shocks can kill even though nothing penetrates the armor!.

The AB-Matter net (which can be designed to be gas-impermeable) may be used for inflatable construction of such strength and lightness as to be able to suspend the weight of a city over a vast span the width of a sea. AB-Matter may also be used for cubic or tower solid construction as it is shown in Fig. 6. 
Estimation and computation of properties of ABmatter:

Strength of AB-matter: Strength (tensile stress) of single string (AB-Matter monofilament). The average connection energy of two nucleons is:

$1 \mathrm{eV}=1.6 \times 10^{-19} \mathrm{~J}, \mathrm{E}=8 \mathrm{MeV}=12.8 \times 10^{-13} \mathrm{~J}$

The average effective distance of the strong force is about $\mathrm{l}=2 \mathrm{fm}=2 \times 10^{-15} \mathrm{~m}\left(1 \mathrm{fm}=10^{-115} \mathrm{~m}\right)$. The average connection force $\mathrm{F}$ the single thread is about:

$\mathrm{F}_{1}=\mathrm{E} / \mathrm{l}=6.4 \times 10^{2} \mathrm{~N}$

This is worth your attention: A thread having diameter 100 thousand times less than an atom's diameter can suspend a weight nearly of human mass. The man may be suspended this invisible and permeable thread(s) and people will not understand how one fly. Specific ultimate tensile stress of single string for cross-section area $\mathrm{s}=2 \times 2=4 \mathrm{fm}^{2}=4 \times 10^{-30} \mathrm{~m}^{2}$ is:

$\sigma=\mathrm{F} / \mathrm{s}=1.6 \times 10^{32} \mathrm{~N} \mathrm{~m}^{-2}$

Compressive stress for $\mathrm{E}=30 \mathrm{MeV}$ and $1=0.4 \mathrm{fm}$ (Fig. 1) is:

$\sigma=\mathrm{E} / \mathrm{sl}=3 \times 10^{33} \mathrm{~N} \mathrm{~m}^{-2}$

The Young's modulus of tensile stress for elongation of break $\varepsilon=1$ is:

$\mathrm{I}=\sigma / \varepsilon=1.6 \times 10^{32} \mathrm{~N} \mathrm{~m}^{-2}$

The Young's modulus of compressive stress for $\varepsilon=0.4$ is:

$\mathrm{I}=\sigma / \varepsilon=7.5 \times 10^{33} \mathrm{~N} \mathrm{~m}^{-2}$

Comparison: Stainless steel has a value of $\sigma=(0.65-$ 1) $\times 10^{9} \mathrm{~N} \mathrm{~m}^{-2}, \quad I=2 \times 10^{11} \mathrm{~N} \mathrm{~m}^{-2}$. Nanotubes has $\sigma=(1.4 \div 5) \times 10^{10} \mathrm{~N} \mathrm{~m}^{-2}, \mathrm{I}=8 \times 10^{11} \mathrm{~N} \mathrm{~m}^{-2}$. That means AB-Matter is stronger by a factor of $10^{23}$ times than steel (by 100 thousands billion by billions times!) and by $10^{22}$ times than nanotubes (by 10 thousand billion by billions times!). Young's modulus and the elastic modulus also are billions of times more than steel and elongation is tens times better than the elongation of steel. Strength (average tensile force) of one $\mathrm{m}$ thin (one layer, $1 \mathrm{fm}$ ) film ( $1 \mathrm{~m}$ compact net) from single strings with step size of grid $1=2 \mathrm{fm}=2 \times 10^{-15} \mathrm{~m}$ is:
$\mathrm{F}=\mathrm{F}_{1} / \mathrm{l}=3.2 \times 10^{17} \mathrm{~N} \mathrm{~m}^{-1}=3.2 \times 10^{13}$ tons $^{-1}$

Strength (average tensile force) of net from single string with step (mesh) size $1=10^{-10} \mathrm{~m}$ (less than a molecule size of conventional matter) which does not pass the any usual gas, liquids or solid (an impermeable net, essentially a film to ordinary matter):

$\mathrm{F}=\mathrm{F}_{1} / \mathrm{l}=6.4 \times 10^{12} \mathrm{~N} \mathrm{~m}^{-1}=6.4 \times 10^{8}$ tons $\mathrm{m}^{-1}$

That means one meter of very thin $(1 \mathrm{fm})$ net can suspend 100 millions tons of load. The tensile stress of a permeable net (it will be considered later) having $1=10^{-7} \mathrm{~m}$ is:

$\mathrm{F}=\mathrm{F}_{1} / \mathrm{l}=6.4 \times 10^{9} \mathrm{~N} \mathrm{~m}^{-1}=6.4 \times 10^{5}$ tons $\mathrm{m}^{-1}$

Specific density and specific strength of $\mathrm{AB}$-matter: The mass of $1 \mathrm{~m}$ of single string (AB-Matter. Monofilament) is:

$\mathrm{M}_{1}=\mathrm{m} \mathrm{L}^{-1}=1.67 \times 10^{-27} /\left(2 \times 10^{-15}\right)=8.35 \times 10^{-13} \mathrm{~kg}$

Where:

$\mathrm{m}=1.67 \times 10^{-27} \mathrm{~kg}$ is mass of one nucleon

$\mathrm{L}=2 \times 10^{-15} \mathrm{~m}$ is distance between nucleons.

The volume of $1 \mathrm{~m}$ one string is $\mathrm{v}=10^{-30} \mathrm{~m}^{3}$.

That means the specific density of AB-Matter string and compact net is:

$\mathrm{d}=\gamma=\mathrm{M}_{1} / \mathrm{v}=8.35 \times 10^{17} \mathrm{~kg} \mathrm{~m}^{-3}$

That is very high (nuclear) specific density. But the total mass is nothing to be afraid of since, the dimensions of $\mathrm{AB}-$ Matter string, film and net are very small and mass of them are:

Mass of string $\mathrm{M}_{1}=8.35 \times 10^{-13} \mathrm{~kg}(\mathrm{see}(10))$

Mass of $1 \mathrm{~m}^{2}$ solid film

$\mathrm{M}_{\mathrm{f}}=\mathrm{M}_{1} / \mathrm{l}=4.17 \times 10^{2} \mathrm{~kg}, 1=2 \times 10^{-15}$

Mass of $1 \mathrm{~m}^{2}$ impenetrable net

$\mathrm{M}_{\mathrm{i}}=\mathrm{M}_{1} \mathrm{~L}^{-1}=8.35 \times 10^{-3} \mathrm{~kg}, \mathrm{~L}=10^{-10} \mathrm{~m}$

Mass of $1 \mathrm{~m}^{2}$ permeable net

$\mathrm{M}_{\mathrm{p}}=\mathrm{M}_{1} \mathrm{~L}^{-1}=8.35 \times 10^{-6} \mathrm{~kg}, \mathrm{l}=10^{-7} \mathrm{~m}$

As you see the fiber, nets from AB-Matter have very high strength and very small mass. To provide an absolute heat shield for the Space Shuttle Orbiter that 
could withstand reentries dozens of times worse than today would take only 100 kilograms of mass for 1105 square meters of surface and the offsetting supports. The specific strength coefficient of AB-Matter-very important in aerospace $-{ }^{[3-5]}$ is:

$$
\begin{aligned}
\mathrm{k}= & \sigma / \mathrm{d}=1.6 \times 10^{32} / 8.35 \times 10^{17}=1.9 \times 10^{14}(\mathrm{~m} \mathrm{sec})^{-2} \\
<\mathrm{c}^{2} & =\left(3 \times 10^{8}\right)^{2}=9 \times 10^{16}(\mathrm{~m} \mathrm{sec})^{-2}
\end{aligned}
$$

This coefficient from conventional high strong fiber has value about $\mathrm{k}=(1-6) \times 10^{9[3-6]}$. AB-Matter is 10 million times stronger. The specific mass and volume density of energy with $\mathrm{AB}-$ Matter are:

$E_{v}=E / v=1.6 \times 10^{32} \mathrm{~J} \mathrm{~m}^{-3}, E_{m}=E / m_{p}=7.66 \times 10^{14} \mathrm{~J} \mathrm{~kg}^{-1}$

Here:

$\mathrm{E}=12.8 \times 10^{-13} \mathrm{~J}$ is (1)

$\mathrm{m}_{\mathrm{p}}=1.67 \times 10^{-27} \mathrm{~kg}$ is nucleon mass $\mathrm{kg}$

$\mathrm{v}=8 \times 10^{-45} \mathrm{~m}^{3}$ is volume of one nucleon

The average specific pressure may reach:

$\mathrm{P}=\mathrm{F}_{1} / \mathrm{s}=12.8 \times 10^{-13} / 4 \times 10^{-30}=3.2 \times 10^{-27} \mathrm{~N} / \mathrm{m}^{2}$

Failure temperature of AB-matter and suitability for thermonuclear reactors: The strong nuclear force is very powerful. That means the outer temperature which must to be reached to destroy the AB fiber, film or net is $\mathrm{T}_{\mathrm{e}}=6 \mathrm{MeV}$. If we transfer this temperature in Kelvin degrees we get:

$\mathrm{T}_{\mathrm{k}}=1.16 \times 10^{4} \mathrm{~T}_{\mathrm{e}}=7 \times 10^{10} \mathrm{~K}$

That temperature is 10 thousands millions degrees. It is about 50-100 times more than temperature in a fusion nuclear reactor. The size and design of the fusion reactor may be small and simple (for example, without big superconductive magnets, cryogenics). We can add the $\mathrm{AB}$ matter has zero heat/thermal conductivity (see later) and it cannot cool the nuclear plasma. This temperature is enough for nuclear reaction of the cheap nuclear fuel, for example, $\mathrm{D}+\mathrm{D}$. The $\mathrm{AB}$ matter may be used in a high efficiency rocket and jet engines, in a hypersonic aircraft and so on. No even in theory can conventional materials have this fantastic thermal resistance!.

Energy generated by production of AB-Matter: Getting of AB-matter produces a large amount of nuclear energy. That energy is more than the best thermonuclear fusion reaction produces. Joining of each nucleon produces $8 \mathrm{MeV}$ energy, when joining the deuterium $\mathrm{D}$ and tritium $\mathrm{T}(2+3=5$ nucleolus $)$ produced only $17.5 \mathrm{MeV}$ (3.6 $\mathrm{MeV}$ for every nucleon). If we use the ready blocks of nucleons as the $\mathrm{D}={ }^{2} \mathrm{H}, \mathrm{T}={ }^{3} \mathrm{H},{ }^{4} \mathrm{He}$ the produced energy decreases. Using the ready nucleus blocks may be necessary because these reactions create the neutrons (n). For example:

${ }^{2} \mathrm{H}+{ }^{2} \mathrm{H} \rightarrow{ }^{3} \mathrm{He}+\mathrm{n}+3.27 \mathrm{MeV},{ }^{3} \mathrm{H}+{ }^{2} \mathrm{H} \rightarrow{ }^{4} \mathrm{He}+\mathrm{n}+17.59 \mathrm{MeV}$ (19)

Which may be useful for producing the needed $\mathrm{AB}$-matter. Using the ready blocks of nucleons decreases the energy getting in $\mathrm{AB}$-Matter production but that decreases also the cost of needed material and enormously simplifies the technology.

A small part $(0.7 \mathrm{MeV})$ of this needed energy will be spent to overcome the Coulomb barrier when the proton joins to proton. Connection of neutrons to neutron or proton does not request this energy (as there is no repulsion of charges). It should be no problem for current technology to accelerate the protons for energy $0.7 \mathrm{MeV}$.

For example, compute the energy in production of $\mathrm{m}=1 \mathrm{~g}=0.001 \mathrm{~kg}$ of AB-matter:

$\mathrm{E}_{1 \mathrm{~g}}=\mathrm{E}_{1} \mathrm{~m} / \mathrm{m}_{\mathrm{p}}=7.66 \times 10^{11} \mathrm{~J} \mathrm{~g}^{-1}$

Here:

$\mathrm{E}_{1}=8 \mathrm{MeV}$ is $12.8 \times 10^{-13}$

$\mathrm{J}=$ Energy produced for joining 1 nucleon

$\mathrm{m}_{\mathrm{p}}=1.67 \times 10^{-27} \mathrm{~kg}$ is mass of nucleon

One $\mathrm{kg}$ of gasoline (benzene) produces $44 \mathrm{MJ} \mathrm{kg}^{-1}$ energy. That means that $1 \mathrm{~g}$ of $\mathrm{AB}-$ Matter requires the equivalent energy of 17.4 tons of benzene.

Super-dielectric strength of AB-matter film: Dielectric strength equals:

$\mathrm{E}_{\mathrm{d}}=\mathrm{E} / \mathrm{l}=8 \mathrm{MV} / 10^{-15} \mathrm{~m}=8 \times 10^{15} \mathrm{MV} / \mathrm{m}$

The best conventional material has dielectric strength of only $680 \mathrm{MV} / \mathrm{m}^{[4]}$.

AB-Matter with orbiting electrons or immersed in electron cloud. We considered early the AB-Matter which contains the electrons within its' own string, film or net. The strong nuclear force keeps the electron (as any conventional matter particle would) in its sphere of influence. But another method of interaction and compensation of electric charges is possible-rotation of electrons around $\mathrm{AB}-$ Matter string (or other linear member) or immersing the $\mathrm{AB}$-Matter string (or other 
linear member, or AB-Matter net-) in a sea of electrons or negative charged atoms (ions). The first case is shown in Fig. 3d, e.g., the second case is shown in Fig. 3f.

The first case looks like an atom of conventional matter having the orbiting electron around the nucleus. However our case has a principal difference from conventional matter. In normal matter the electron orbits around the nucleus as a POINT. In our case it orbits around the charged nuclear material (AB-Matter) LINE (some form of linear member from AB-Matter). That gives a very important difference in electrostatic force acting on the electron. In conventional cases (normal molecular matter) the electrostatic force decreases as $1 / \mathrm{r}^{2}$, in our AB-Matter case the electrostatic force decreases as $1 / \mathrm{r}$. The interesting result (see below) is that the electron orbit in $\mathrm{AB}$ Matter does follow the usual speed relationship to radius. The proof is below:

$$
\begin{aligned}
\frac{\mathrm{mV}^{2}}{\mathrm{r}} & =\mathrm{eE}, \quad \mathrm{E}=\mathrm{k} \frac{2 \tau}{\mathrm{r}}, \quad \mathrm{mV}^{2}=2 \mathrm{k} \tau \mathrm{e}, \quad \mathrm{V}=\sqrt{\frac{2 \mathrm{ke} \tau}{\mathrm{m}}} \\
& =\sqrt{\mathrm{N}_{\mathrm{p}}} \mathrm{e} \sqrt{\frac{2 \mathrm{k}}{\mathrm{m}}}=22.4 \sqrt{\mathrm{N}_{\mathrm{p}}}
\end{aligned}
$$

Where:

$\mathrm{m}=\mathrm{m}_{\mathrm{e}}=9.11 \times 10^{-31} \mathrm{~kg}$

$\mathrm{V}=$ Electron speed $\mathrm{m} \mathrm{sec}^{-1}$

$\mathrm{r}=$ Radius of electron orbit $\mathrm{m}$

$\tau=$ Charge density in $1 \mathrm{~m}$ of single string $\mathrm{C} / \mathrm{m}$

$\mathrm{E}=$ Electrostatic intensity $\mathrm{A} / \mathrm{m}$ or $\mathrm{N} / \mathrm{C}$

$\mathrm{k}=9 \times 10^{9} \mathrm{Nm}^{2} / \mathrm{C}^{2}$ is electrostatic constant

$\mathrm{e}=1.6 \times 10^{-19} \mathrm{C}$ is charge of electron $\mathrm{C}$

$\mathrm{N}_{\mathrm{p}}=$ Number of proton in $1 \mathrm{~m}$ of single string, $1 / \mathrm{m}$

As you see from last Eq. 22 the electron speed is not relative to radius. The real speed will be significantly less than given Eq. 22 because the other electrons block the charge of the rest of the string. The total charge of the system is zero. Therefore we can put $\mathrm{N}_{\mathrm{p}}=1$ (every electron in orbit is kept by only one proton in string). From last Eq. 22 we find $V=22.4 \mathrm{~m} \mathrm{sec}^{-1}$. That means the electron speed carries only a very small energy.

In the second case the AB-Matter (string girder) can swim in a cloud (sea) of electrons. That case occurs in metals of conventional matter. But a lattice of metallic ions fills the volume of conventional metal giving drag to electron flow (causing electrical resistance).

The stringers and plate nets of AB-Matter can locate along the direction of electric flow. They constitute only a relatively tiny volume and will produce very small electric resistance. That means the
AB-Matter may be quasi-super-conductivity or superconductivity. The electrons rotate around an ABMatter string repel one from other. The tensile force from them is:

$$
\begin{aligned}
\mathrm{F} & =\mathrm{k} \frac{\mathrm{e}^{2}}{\mathrm{~d}^{2}}\left(1+\frac{1}{2^{2}}+\frac{1}{3^{2}}+\ldots+\frac{1}{\mathrm{n}^{2}}+\ldots\right) \\
& =\frac{\pi^{2} \mathrm{k}}{6} \frac{\mathrm{e}^{2}}{\mathrm{~d}^{2}}=1.476 \cdot 10^{10} \frac{\mathrm{e}^{2}}{\mathrm{~d}^{2}}
\end{aligned}
$$

For distance $\mathrm{d}=2 \times 10^{-15} \mathrm{~m}$ the force equals $\mathrm{F}=10.5 \mathrm{~N}$. This force keeps the string and net in unfolded stable form.

Some properties of AB-matter: We spoke about the fantastic tensile and compressive strength, rigidity, hardness, specific strength, thermal (temperature) durability, thermal shock and big elongation of $\mathrm{AB}$ Matter.

Short note about other miraculous AB-Matter properties:

- Zero heat/thermal capacity. That follows because the mass of nucleons (AB-Matter string, film, net) is large in comparison with mass single atom or molecule and nucleons in AB-Matter have a very strong connection one to other. Conventional atoms and molecules cannot pass their paltry energy to AB-Matter! That would be equivalent to moving a huge dry-dock door of steel by impacting it with very light table tennis balls Zero heat/thermal conductivity

- Absolute chemical stability. No corrosion, material fatigue. Infinity of lifetime. All chemical reactions are acted through ORBITAL electron of atoms. The AB-Matter does not have orbital electrons (special cases will be considered later on). Nucleons cannot combine with usual atoms having electrons. In particular, the AB-Matter has absolute corrosion resistance. No fatigue of material because in conventional material fatigue is result of splits between material crystals. No crystals in ABMatter. That means AB-Matter has lifetime equal to the lifetime of neutrons themselves. Finally a container for the universal solvent!

- Super-transparency, invisibility of special ABMatter-nets. An AB-Matter net having a step distance (mesh size) between strings or monofilaments of more than $100 \mathrm{fm}=10^{-13} \mathrm{~m}$ will pass visible light having the wave length (400$800) \times 10^{-9} \mathrm{~m}$. You can make cars, aircraft and space ships from such a permeable (for visible 
light) AB-Matter net and you will see a man (who is made from conventional matter) apparently sitting on nothing, traveling with high speed in atmosphere or space without visible means of support or any visible vehicle!

- Impenetrability for gas, liquids and solid bodies. When the AB-Matter net has a step size between strings of less than atomic size of $10^{-10} \mathrm{~m}$, it became impenetrabile for conventional matter. Simultaneously it may be invisible for people and have gigantic strength. The AB-Matter net may-as armor--protect from gun, cannon shells and missiles

- Super-impenetrability for radiation. If the cell size of the AB-Matter net will be less than a wave length of a given radiation, the AB-Matter net does not pass this radiation. Because this cell size may be very small, $\mathrm{AB}$ net is perfect protection from any radiation up to soft gamma radiation (include radiation from nuclear bomb)

- Full reflectivity (super-reflectivity). If the cell size of an AB-Matter net will be less than a wavelength of a given radiation, the $\mathrm{AB}$-Matter net will then fully reflect this radiation. With perfect reflection and perfect impenetrability remarkable optical systems are possible. A Fresnel like lens might also be constructible of AB-Matter

- Permeable property (ghost-like intangibility power; super-passing capacity). The AB-Matter net from single strings having mesh size between strings of more than $100 \mathrm{~nm}=10^{-11} \mathrm{~m}$ will pass the atoms and molecules through itself because the diameter of the single string $\left(2 \times 10^{-15} \mathrm{~m}\right)$ is 100 thousand times less then diameter of atom $\left(3 \times 10^{-10} \mathrm{~m}\right)$. That means that specifically engineered constructions from AB-Matter can be built on the Earth, but people will not see and feel them. The power to phase through walls, vaults and barriers has occasionally been portrayed in science fiction but here is a real life possibility of it happening

- Zero friction. If the $\mathrm{AB}-M a t t e r$ net has a mesh size distance between strings equals or less to the atom $\left(3 \times 10^{-10} \mathrm{~m}\right)$, it has an ideal flat surface. That means the mechanical friction may be zero. It is very important for aircraft, sea ships and vehicles because about $90 \%$ of its energy they spend in friction. Such a perfect surface would be of vast value in optics, nanotech molecular assembly and prototyping, physics labs

- Super or quasi-super electric conductivity at any temperature. As it is shown in previous section the AB-Matter string can have outer electrons in an arrangement similar to the electronic cloud into metal. But AB-Matter strings (threads) can be located along the direction of the electric intensity and they will not resist the electron flow. That means the electric resistance will be zero or very small

- High dielectric strength (Eq. 21)

- AB-Matter may be used for devices to produce high magnetic intensity

Some applications of AB-matter: The applications of the AB-Matter are encyclopedic in scope. This matter will create revolutions in many fields of human activity. We show only non-usual applications that come to mind and by no means all of these:

- Storage of gigantic energy. As it is shown in ${ }^{[3-6]}$, the energy saved by flywheel equals the special mass density of material (17). As you see that is a gigantic value of stored energy because of the extreme values afforded by the strong nuclear force. Car having a pair of 1 gram counterspun flywheels ( $2 \mathrm{~g}$ total) (20) charged at the factory can run all its life without benzene. Aircraft or sea ships having $100 \mathrm{~g}$ (two $50 \mathrm{~g}$ counterspun flywheels) can fly or swim all its life without additional fuel. The offered flywheel storage can has zero friction and indefinite energy storage time

- AB-Matter as propulsion system of space ship. The most important characteristic of rocket engine is specific impulse (speed of gas or other material flow out from propulsion system). Let us compute the speed of a part of fly-wheel ejected from the offered rocket system:

$\frac{\mathrm{mV}^{2}}{2}=\mathrm{E}, \quad \mathrm{V}=\sqrt{\frac{2 \mathrm{E}}{\mathrm{m}}}=3.9 \cdot 10^{7} \mathrm{~m} \mathrm{sec}^{-1}$

Here:

$\mathrm{V}=$ Speed of nucleon $\mathrm{m} \mathrm{sec}^{-1}$

$\mathrm{E}=12.8 \times 10^{-13} \mathrm{~J}(1)$ is energy of one nucleon $\mathrm{J}$

$\mathrm{M}=1,67 \times 10^{-27} \mathrm{~kg}$ is mass of one nucleon, $\mathrm{kg}$

The value (24) is about $13 \%$ of light speed.

The chemical rocket engine has specific impulse about $3700 \mathrm{~m} \mathrm{sec}^{-1}$. That value is 10 thousand times less. The electric rocket system has a high specific impulse but requires a powerful compact and light source of energy. In the offered rocket engine the energy is saved in the flywheel. The current projects of a nuclear rocket are very complex, heavy and dangerous for men (gamma and neutron radiation) and 
have specific impulse of thousand of times less (24). The offered AB-Matter rocket engine may be very small and produced any rocket thrust in any moment in any direction.

Super-weapon: Capability of an AB-Matter flywheel to spin up and ejection matter at huge speed (24) may be used as a long distance super-weapon.

Super-armor from conventional weapons: The value (24) gives the need speed for break through (perforation) of a shield of AB-Matter. No weapon which can give this speed exists at the present time. Remain, the AB-Matter may be radiation impermeable. That means AB-Matter can protect from a nuclear bomb and laser weapon.

Simple thermonuclear reactor: The AB-Matter film may be used as the wall of a simple thermonuclear reactor. The AB-Matter film allows a direct $100 \%$ hit by the accelerated nuclei to stationary nuclei located into film. You get a controlled nuclear reaction of cheap fuel. For example:

${ }^{1} \mathrm{H}+{ }^{1} \mathrm{H} \rightarrow{ }^{2} \mathrm{H}+\mathrm{e}^{+}+\mathrm{v}+0.42 \mathrm{MeV}$,

${ }^{2} \mathrm{H}+{ }^{1} \mathrm{H} \rightarrow{ }^{3} \mathrm{He}+\gamma+5.494 \mathrm{MeV}$

${ }^{2} \mathrm{H}+{ }^{2} \mathrm{H} \rightarrow{ }^{3} \mathrm{H}+{ }^{1} \mathrm{H}+4.033 \mathrm{MeV}$,

${ }^{3} \mathrm{H}+{ }^{1} \mathrm{H} \rightarrow{ }^{4} \mathrm{He}+\gamma+16.632 \mathrm{MeV}$

Here:

$$
\begin{aligned}
\mathrm{e}^{+} & =\text {Electron } \\
\mathrm{v} & =\text { Neutrino } \\
\gamma & =\gamma \text {-quantum, photon }(\gamma \text {-radiation }) \\
{ }^{1} \mathrm{H} & =\mathrm{p}=\text { proton } \\
{ }^{2} \mathrm{H} & =\mathrm{D}=\text { deuterium } \\
{ }^{3} \mathrm{H} & =\mathrm{T}=\text { tritium } \\
\mathrm{He} & =\text { Helium }
\end{aligned}
$$

In conventional thermonuclear reactor the probability of a hit by the accelerated (or highly heated) nuclei to other nuclei is trifling. The accelerated particles, which run through ghostlike ATOMS and lose the energy, need therefore to be sent through to repeated collisions each of which loses energy until the one that hits and generates energy. The winner must pay for all the losers. That way we need big, very complex and expensive high temperature conventional thermonuclear reactors. They are so nearly unbuildable because ordinary matter literally cannot take the reactions they are designed to contain and therefore special tricks must be used to sidestep this and the reactions are so improbable that again special tricks are required. Here, every shot is a hit and the material can endure every consequence of that hit. A good vacuum system and a means of getting power and isotopes in and out are the main problems and by no means insuperable ones. Using the AB-Matter we can design a microthermonuclear $\mathrm{AB}$ reactor.

High efficiency rocket, jet and piston aviation engines: The efficiency conventional jet and rocket engines are very limited by the temperature and safety limits of conventional matter $\left(2000^{\circ} \mathrm{K}\right)$. If we will design the rotor blades (in jet engine), combustion chamber (in rocket and piston engines) from ABMatter, we radically improve their capacities and simplify their construction (for example, no necessary cooling system!).

Hypersonic aircraft: The friction and heat which attacks conventional materials for hypersonic aircraft limits their speed. Using the AB-Matter deletes this problem. Many designs for aerospace planes could capture oxygen in flight, saving hauling oxidizer and carrying fuel alone-enabling airliner type geometries and payloads since the weight of the oxidizer and the tanks needed to hold it and the airframe strengths required escalate the design and cascade through it until conventional materials today cannot build a single stage to orbit or antipodes aerospace plane. But that would be quite possible with AB-Matter.

Increasing efficiency of a conventional aviation and transport vehicles: AB-Matter does not experience friction. The air drag in aviation is produced up $90 \%$ by air friction on aircraft surface. Using AB-Matter will make jump in flight characteristics of aircraft and other transport vehicles (including sea ships and cars).

Improving capabilities of all machines: Appearance new high strength and high temperature AB-Matter will produce jump, technology revolution in machine and power industry.

Computer and computer memory: The AB-Matter film allows to write in $1 \mathrm{~cm}^{2} \mathrm{~N}=1 /\left(4 \times 10^{-26}\right)=$ $2.5 \times 10^{25} 1 \mathrm{~cm}^{-2}$ bits information. The current 45 nanometer technology allows to write only $\mathrm{N}=2.5 \times 10^{14}$ $1 \mathrm{~cm}^{-2}$ bit. That means the main chip and memory of computer based in AB-Matter film may be a billion times smaller and presumably thousands of times faster (based on the lesser distance signals must travel). The reader can imagine useful application of AB-Matter in any field he is familiar with. 


\section{RESULTS AND DISCUSSION}

\section{Pauli exclusion principle and Heisenberg uncertainty principle:}

General question of stability: The reader may have questions about compatibility of the Pauli exclusion principle and Heisenberg Uncertainty Principle with AB-Matter. The uncertainty principle is:

$\Delta \mathrm{p} \Delta \mathrm{x} \geq \hbar / 2$

Where:

$\Delta \mathrm{p}=\mathrm{mV}$ is momentum of particle $\mathrm{kg} \mathrm{m} \mathrm{sec}{ }^{-1}$

$\mathrm{m}=$ Mass particles $\mathrm{kg}$

$\mathrm{V}=$ Speed particles $\mathrm{kg} \mathrm{m} \mathrm{sec}{ }^{-1}$

$\Delta \mathrm{x}=$ Distance between particles $\mathrm{m}$

$\hbar=6.6262 \times 10^{-34} / 2 \pi$ is Planck's constant

Pauli states that no two identical fermions may occupy the same quantum state simultaneously. A more rigorous statement of this principle is that, for two identical fermions, the total wave function is antisymmetric. For electrons in a single atom, it states that no two electrons can have the same four quantum numbers, that is, if particles characteristics $\mathrm{n}, 1$ and $\mathrm{m}_{1}$ are the same, $\mathrm{m}_{\mathrm{s}}$ must be different such that the electrons have opposite spins.

The uncertainty principle gives a high uncertainty of $\Delta \mathrm{p}$ for nucleons and very high uncertainty for electrons into AB-Matter. But high density matter (of the same order as our suggested AB-Matter) EXISTS in the form of nuclei of conventional matter and on neutron stars. That is an important proof-this matter exists. Some may question its' ability to stay in a superdense state passively. Some may doubt its' stability free of the fierce gravitation of neutron stars (natural degenerate matter) or outside the confines of the nucleus. But there are reasons, not all stated here, to suppose that it might be so stable under normal conditions.

One proof was provided by Freeman Dyson ${ }^{[11]}$ and Andrew Lenard in 1967, who considered the balance of attractive (electron-nuclear) and repulsive (electronelectron and nuclear-nuclear) forces and showed that ordinary matter would collapse and occupy a much smaller volume without the Pauli principle. Certainly, however this very question of stability will be a key focus of any detailed probe into the possibilities of $\mathrm{AB}$ Matter.

\section{Micro-world from AB-matter:}

An amusing thought-experiment: AB-Matter may have $10^{15}$ times more particles in a given volume than a single atom. A human being, man made from conventional matter, contains about $5 \times 10^{26}$ molecules. That means that 200 'femto-beings' of equal complexity from AB-Matter (having same number of components) could be located in the volume of one microbe having size $10 \mu=10^{-5} \mathrm{~m}$. If this proved possible, we could not see them, they could not see us in terms of direct sensory input. Because of the wavelength of light it is questionable what they could learn of the observable macro-Universe. The implications, for transhuman scenarios, compact interstellar (microbe sized!) payloads, uploading and other such scenarios are profound. It is worth recalling that a single house and garden required to support a single conventional matter human is, for AB-Matter 'femto-beings', equivalent in relative vastness as the extended Solar system is for us. If such a future form could be created and minds 'uploaded' to it, the future theoretical population, knowledge base and scholarly and knowledge-industries output of even a single planet so populated could rival that of a theoretical Kardashev Type III galactic civilization!

Note: The same idea may hypotheticaly be developed for atto $\left(10^{-18} \mathrm{~m}\right)$, zepto $\left(10^{-21} \mathrm{~m}\right)$ and yocto $\left(10^{-24} \mathrm{~m}\right)$ technologies. It is known that nucleons consist of quarks. Unfortunately, we do not know yet about size, forces and interactions between quark and cannot therefore make predictions about atto or zeptotechnology. One theory posits that the quark consists of preons. But we do not know anything about preons. The possibility alone must intrigue us for now. Where does it all end?

\section{CONCLUSION}

The researcher offers a design for a new form of nuclear matter from nucleons (neutrons, protons), electrons and other nuclear particles. He shows that the new AB-Matter has most extraordinary properties (for example, (in varying circumstances) remarkable tensile strength, stiffness, hardness, critical temperature, superconductivity, super-transparency, ghostlike ability to pass through matter, zero friction), which are millions of times better than corresponded properties of conventional molecular matter. He shows how to design aircraft, ships, transportation, thermonuclear reactors and constructions and so on from this new nuclear matter. These vehicles will have correspondingly amazing possibilities (invisibility, passing through any walls and amour, protection from nuclear bombs and any radiation).

People may think this fantasy. But fifteen years ago most people and many scientists thought- 
Am. J. Engg. \& Applied Sci., 2 (2): 501-514, 2009

nanotechnology ${ }^{[10]}$ is fantasy. Now many groups and industrial labs, even startups, spend hundreds of millions of dollars for development of nanotechnological-range products (precise chemistry, patterned atoms, catalysts, metamaterials) and we have nanotubes (a new material which does not exist in Nature!) and other achievements beginning to come out of the pipeline in prospect. Nanotubes are stronger than steel by a hundred times-surely an amazement to a 19th Century observer if he could behold them. Nanotechnology, in near term prospect, operates with objects (molecules and atoms) having the size in nanometer $\left(10^{-9} \mathrm{~m}\right)$. The author here outlines perhaps more distant operations with objects (nuclei) having size in the femtometer range, $\left(10^{-15} \mathrm{~m}\right.$, millions of times less smaller than the nanometer scale). The name of this new technology is femtotechnology.

I want to explain the main thrust of this by analogy. Assume we live some thousands of years ago in a great river valley where there are no stones for building and only poor timber. In nature we notice that there are many types of clay (nuclei of atom-types of element). One man offers to people to make from clay bricks (AB-Matter) and build from these bricks a fantastic array of desirable structures too complex to make from naturally occurring mounds of mud. The bricks enable by increased precision and strength things impossible before. A new level of human civilization begins.

I call upon scientists and the technical community to research and develop femtotechnology. I think we can reach in this field progress more quickly than in the further prospects of nanotechnology, because we have fewer (only 3) initial components (proton, neutron, electron) and interaction between them is well-known (3 main forces: Strong, weak, electrostatic). The different conventional atoms number about 100, most common molecules are tens thousands and interactions between them are very complex (e.g., Van der Waals force). It may be however, that nano and femto technology enable each other as well, as tiny bits of AB-Matter would be marvelous tools for nanomechanical systems to wield to obtain effects unimaginable otherwise. What time horizon might we face in this quest? The physicist Richard Feynman offered his idea to design artificial matter from atoms and molecules at an American Physical Society meeting at Caltech on December 29, 1959. But only in the last 15 years we have initial progress in nanotechnology. On the other hand progress is becoming swifter as more and better tools become common and as the technical community grows. Now are in the position of trying to progress from the ancient 'telega' haywagon of rural Russia (in analogy, conventional matter composites) to a 'luxury sport coupe' (advanced tailored nanomaterials). The researcher suggests we have little to lose and literal worlds to gain by simultaneously researching how to leap from 'telega' to 'hypersonic space plane'. (Femotech materials and technologies, enabling all the wonders outlined here).

\section{ACKNOWLEDGEMENT}

The researcher wishes to acknowledge Joseph Friedlander (of Shave Shomron, Israel) for correcting the English and offering useful advices and suggestions.

\section{REFERENCES}

1. Bolonkin, A.A., 1983a. Method of a keeping of a neutral plasma and installation for it. Russian Patent Application \#3600272/25vv086993, 6 June 1983 (in Russian), Russian PTO.

2. Bolonkin, A.A., 1983b. Method of transformation of plasma energy in electric current and installation for it. Russian Patent Application \#3647344/136681, 27 July 1983 (in Russian), Russian PTO.

3. Bolonkin, A.A., 2006. Non-Rocket Space Launch and Flight. Elsevier, London, ISBN: 0080447317, pp: 468.

4. Bolonkin, A.A., 2007. New Concepts, Ideas, Innovations in Aerospace, Technology and Human Science. NOVA., ISBN: 9781600217876, pp: 509.

5. Bolonkin, A.A. and R.B. Cathcart, 2008. MacroProjects in Environment and Technology. NOVA., ISBN: 13: 9781604569988, pp: 500.

6. Bolonkin, A.A., 2007. Human Immortality and Electronic Civilization. 3rd Edn., Lulu, English Version, pp: 60. \$9.9; Russian version 101 pgs, \$9.9. http://www.lulu.com search "Bolonkin". Old vertion is in http://Bolonkin.narod.ru/p101.htm .

7. Encyclopedia of Physics. http://www.physicum.narod.ru (in Russian). 5: 670, 5: 655 .

8. I.K. Kikoin, 1976. Tables of Physical Values. Reference Book, Moscow, pp: 1006.

9. Cohen, E.R., D.R. Lide, G.L. Trigg, 2003. AIP Physics Desk Reference. 3rd Edition, Springer, ISBN: 0387989730, pp: 888.

10. Dresselhaus, M.S., 2000. Carbon Nanotubes. Springer.

11. Wikipedia, Some background material in this article is gathered from wikipedia under the creative commons license. http://wikipedia.org 